\title{
Effects of appropriate-intensity treadmill exercise on skeletal muscle and respiratory functions in a rat model of emphysema
}

\author{
Hidetaka Imagita ${ }^{1}$, Yasue NishiI ${ }^{1}$, Naoto Fujita ${ }^{2}$, Taiko Sukedzane ${ }^{1}$, and Shinnosuke Kawata ${ }^{1}$ \\ ${ }^{1}$ Graduate School of Health Sciences, Kio University, Nara, Japan and ${ }^{2}$ Graduate School of Health Sciences, Hiroshima University, \\ Hiroshima, Japan
}

(Received 16 August 2019; and accepted 28 November 2019)

\begin{abstract}
The number of patients with chronic obstructive pulmonary disease (COPD), a typical respiratory disorder, is rapidly increasing globally. The purpose of this study was to determine the effects of appropriate-intensity treadmill exercise on skeletal muscle and respiratory functions in a rat model of emphysema. Twenty-one Wistar rats were randomly divided into three groups: the sham (SH) group, pulmonary emphysema (PE) group, and emphysema + exercise (EX) group. Cigarette smoke solution and lipopolysaccharide were intratracheally administered for 4 weeks in the PE and EX groups. The rats in the EX group were made to run on treadmills in the latter 2 weeks of the experiment. Lung tissue was stained with anti-macrophage antibodies; the specific force (contractile force per unit cross-sectional area) of the diaphragm and hind-limb muscles was measured, and blood was analyzed for serum cytokine levels. Many macrophages were observed in the lung tissue of the PE group. In the EX group, the population of macrophages was smaller, and the specific force of the diaphragm and extensor digitorum longus muscles was higher than in the PE group. Moreover, the degree of inflammation in the pulmonary tissue was reduced in the EX group. These results suggest that adaptive exercise may improve not only respiratory and muscle functions but also inflammation of the pulmonary tissue associated with emphysema.
\end{abstract}

The Global Initiative for Chronic Obstructive Lung Disease (13), an international guideline, defines chronic obstructive pulmonary disease (COPD) as "a common preventable and treatable disease, which is characterized by persistent airflow limitation that is usually progressive and associated with an enhanced chronic inflammatory response in the airways and the lung to noxious particles or gases." Patients with COPD, which is a typical respiratory disorder, are rapidly increasing globally, and by 2020 , the disease is expected to become the third most frequent cause of death, following heart disease and stroke (5). The

Address correspondence to: Hidetaka Imagita, Graduate School of Health Sciences, Kio University, 4-2-2 Umami-naka, Koryo-cho, Kitakatsuragi-gun, Nara 635-0832, Japan

Tel: +81-745-54-1601, Fax: +81-745-54-1600

E-mail: h.imagita@kio.ac.jp
Japan COPD Epidemiology study in 2001 reported that the number of potential COPD patients was as high as 5.3 million (10).

The predominant treatment and management of COPD patients include smoking cessation (7); pharmacological management, such as with anticholinergic drugs (8), $\beta 2$ adrenoceptor stimulants (23), and inhaled steroid therapy; therapeutic exercise to recover activities of daily living and quality of life (11, 17,32 ); and nutritional therapy to supplement inadequate intake and increased resting energy expenditure $(6,20,36)$.

There are wide varieties of respiratory disorders, including allergic, infectious, and tumorous lung diseases, in addition to COPD, that not only adversely affect ventilatory function but also exacerbate perceived difficulty in breathing and markedly restrict activities of daily living $(29,34)$. These diseases have also been thought to cause functional abnormalities, 


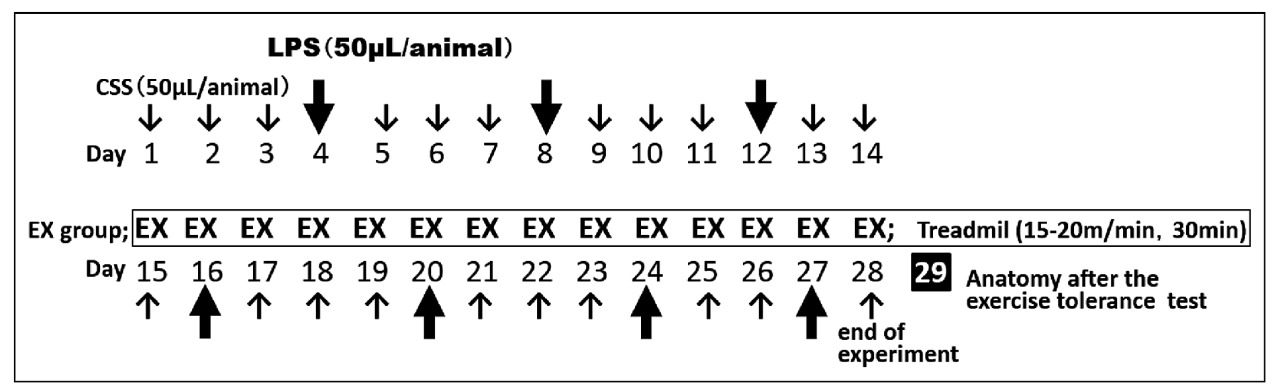

Fig. 1 Schedule for Intratracheal administrations of CSS and LPS in PE group and Ex group. Cigarette smoke solution (CSS), lipopolysaccharide (LPS), or saline was intratracheally administered once per day for 4 weeks with the use of inhalant anesthetics. The exercise group was also made to run on treadmills in the latter 15 days of the experiment.

such as decreased contraction force and endurance capacity of skeletal muscles, and easy fatigability. Skeletal muscle dysfunction can be caused by such conditions of disuse as prolonged immobility and cast immobilization; however, it can also occur from hypoxic stress, hypercapnia, proinflammatory cytokines, oxidant stress, steroid hormones, and so on $(21,35)$. Muscle function can be maintained or improved by appropriate exercise, and it is reported that appropriate exercise also improves immunity (19). On the other hand, exercise is reported to increase proinflammatory cytokine and C-reactive protein levels, which can lead to an inflammatory response to high-intensity exercise $(26,31)$.

The purpose of this study was to determine the influence of appropriate-intensity treadmill running on muscle and respiratory function in a rat model of emphysema. We hypothesized that appropriate-intensity exercise would improve the function of muscle and respiratory, inflammation, and plasma cytokine levels in the rats.

\section{MATERIALS AND METHODS}

Animals. Twenty-one 9-week-old male Wistar rats (body weight, $266.5 \pm 6.2 \mathrm{~g}$ ) were obtained for this study. After one week of installation, the experiment was begun. The temperature and humidity of the animal holding room were maintained at $23^{\circ} \mathrm{C}$ and $50 \%$, respectively, with a 12-h light/dark cycle. Throughout the rearing period, rats had free access to food and water. However, 2 rats were excluded because they died during the experimental period. This experiment was conducted with the approval of the Kio University Animal Experiment Ethical Review Board and in accordance with the Kio University Animal Experiment Management Regulations.

Preparation of the emphysema model rats. We in- duced a rat model of emphysema by administering cigarette smoke solution (CSS) directly into the trachea (22). Animals were randomly divided into three groups: the sham $(\mathrm{SH})$ group, the pulmonary emphysema (PE) group, and the emphysema + exercise (EX) group. The CSS and lipopolysaccharide (LPS; Sigma, St. Louis, MO, USA) were intratracheally administered for 4 weeks to the PE and EX groups, and saline was intratracheally administered to the SH group for the same period. By using an air pump to generate suction, the CSS was prepared by bubbling a stream of smoke into saline $(40 \mathrm{~mL} / 40$ cigarettes). It took approximately $3 \mathrm{~min}$ to bubble the smoke from one cigarette into the saline. The CSS that was diluted by 10 times registered 1.205 at the absorbance of $267 \mathrm{~nm}$ on spectrophotometry. The CSS that was not diluted was administered to the rat by atomization in the upper part of the airway using a hydraulic sprayer (Penn Century).

Research design. The CSS (50 $\mu \mathrm{L} /$ animal) was intratracheally administered once per day on Days 1-3, $5-7,9-11,13-15,17-19,21-23,25,26$, and 28 with the use of inhalant anesthetics (Fig. 1). Subsequently, a LPS solution $(1 \mathrm{mg} / \mathrm{mL} \mathrm{CSS}, 50 \mu \mathrm{L} / \mathrm{ani}$ mal) was intratracheally administered on Days 4,8 , $12,16,20,24$, and 27. The rats in the EX group were made to run on treadmills beginning on Day 15 of the experiment. With respect to exercise intensity, exercise tolerance was tested on Day 15, because high-intensity exercise can cause an inflammatory response. The exercise tolerance test was carried out by a treadmill at a multistep speed from 5 to $20 \mathrm{~m} /$ min for $20 \mathrm{~min}$. Blood lactate levels were measured immediately before exercise, and at 5-, 10-, 15- and 20-min intervals during exercise. The initial exercise intensity was set to $15 \mathrm{~m} / \mathrm{min}$ for $20 \mathrm{~min}$; the load gradually increased afterward, until it was performed at $20 \mathrm{~m} / \mathrm{min}$ for $30 \mathrm{~min}$ on Day 28 . 
All animals in the $\mathrm{SH}$ group, the PE group, and the EX group completed the exercise tolerance test $(20 \mathrm{~m} / \mathrm{min}$ for $30 \mathrm{~min})$ on Day 29, and blood was collected before exercise and $90 \mathrm{~min}$ post-exercise.

\section{Measurement items}

a) Respiratory function measurements. Animals were administered anesthetics by pentobarbital intraperitoneal injection $(1 \mathrm{~mL} / \mathrm{kg} \times$ body weight $) 90 \mathrm{~min}$ following exercise on Day 29, and they were fixed in the supine position. After anesthetizing the animals, tracheotomy was performed, and the inserted cannula was connected to a basic medical research system (LEG-1000; Nihon Kohden, Tokyo, Japan) through a pressure gradient transducer (TP-602T; Nihon Kohden) and respiratory flow meter (RY111S; Nihon Kohden). The respiratory flow waveforms shown on a computer display were recorded, and the tidal volume and inspiration/expiration ratio were calculated from the measured respiratory waves using integration.

b) In vitro measurement of muscle-specific force. The right soleus muscle (soleus), extensor digitorum longus muscle (EDL) and diaphragm muscle (diaphragm) were isolated after respiratory function measurements. The distal end of the excised all muscles were fixed with a clip, the proximal end was connected to an isometric transducer (TB-651T; Nihon Kohden), and the muscle sample was immersed in Ringer's solution, which was continuously aerated with $95 \% \mathrm{O}_{2}: 5 \% \mathrm{CO}_{2}$ and maintained at a $\mathrm{pH}$ of 7.4 and $37^{\circ} \mathrm{C}$. After connecting the sample to the transducer, the muscle was stimulated electrically using plate electrodes, and an optimal muscle length that produced the maximum single-twitch force was determined. The stimulation intensity was set at $125 \%$ of the intensity that produced the maximum single twitch. Based on a twitch curve obtained using a digital oscilloscope, the maximum potential difference, contraction time, half relaxation time and tetanic force were measured at stimulation frequencies of $100 \mathrm{~Hz}$. To obtain muscle-specific force characteristics, we obtained the single-twitch force per unit cross-sectional area at a stimulation frequency of $1 \mathrm{~Hz}$.

c) Immunohistochemistry. After muscle contraction force was measured, lung tissue was fixed by left ventricle instillation with $4 \%$ phosphate buffered paraformaldehyde (PFA). For fixation, lung tissue was placed in 4\% PFA for $1 \mathrm{~h}$ and then intergraded in $10 \%$ to $30 \%$ sucrose solutions overnight at $4^{\circ} \mathrm{C}$. Fixed samples were then frozen in OCT compound. Frozen samples were cut into $20-\mu \mathrm{m}$ thick cross-sections by a Leica CM1850 cryostat. The sections were stained with hematoxylin and eosin for histological observation. In addition, the sections were fixed in acetone at $-20^{\circ} \mathrm{C}$ and blocked with Blocking One Histo (Nakarai Tesque, Kyoto, Japan) for immunofluorescence.

The sections were then incubated at $4^{\circ} \mathrm{C}$ overnight with mouse anti-rat CD68 (1:200, sc-59103; Santa Cruz Biotechnology) antibody to identify total macrophages and double-stained with rabbit anti-human CD206 (1 : 100, sc-48758; Santa Cruz Biotechnology) or rabbit anti-mouse CD163 (1:100, sc33560; Santa Cruz Biotechnology) antibodies to identify M2 macrophages. Subsequently, sections were exposed to Alexa Fluor 488- or 555-conjugated anti-rabbit or anti-mouse immunoglobulin $\mathrm{G}$ ( $1: 2,000$; Cell Signaling, MA, USA) for $60 \mathrm{~min}$ at room temperature, then mounted with a medium containing 4',6-diamidino-2-pheny-lindole (DAPI; H-1500, Vector Laboratories). Parallel slides processed without the primary antibody served as negative controls. The sections were analyzed, and images were acquired on a fluorescent microscope (Eclipse 80i; Nikon, Tokyo, Japan). The numbers of CD68 ${ }^{+}$, $\mathrm{CD} 68^{+} / \mathrm{CD} 206^{+}$, and $\mathrm{CD} 68^{+} / \mathrm{CD} 163^{+}$cells per DAPIpositive nuclei were quantified in four fields per lung tissue. All measurements were performed with Image J software (NIH).

d) Inflammatory cytokines. Blood samples were collected immediately before exercise and at $90 \mathrm{~min}$ post-exercise on Day 29. Blood samples were drawn from the cervical vein with uncoated tubes. After centrifugation, plasma was stored at $-80^{\circ} \mathrm{C}$. Serum samples were analyzed in duplicate for inflammatory cytokines with a bead-based multiplex assay using the Bio-Plex instrument and xPONENT analysis software (Luminex, Austin, TX). Interleukin (IL)-1 $\beta$, IL-4, IL-6, IL-10, and tumor necrosis factor (TNF)- $\alpha$ were measured using a commercially available assay kit (Bio-rad, Billerica, MA) according to the manufacturer's specifications.

Statistical Analysis. Data are expressed as means \pm standard deviation. A one-way ANOVA was used to compare differences between groups, and multiple comparisons were made using Sheffe's method. Regarding the blood lactate level and inflammatory cytokines before and after exercise on Day 29, we calculated the before-and-after exercise ratio and used nonparametric tests (Kruskal-Wallis and SteelDwass tests) to compare differences between groups. Statistical significance was set at $P<0.05$. 


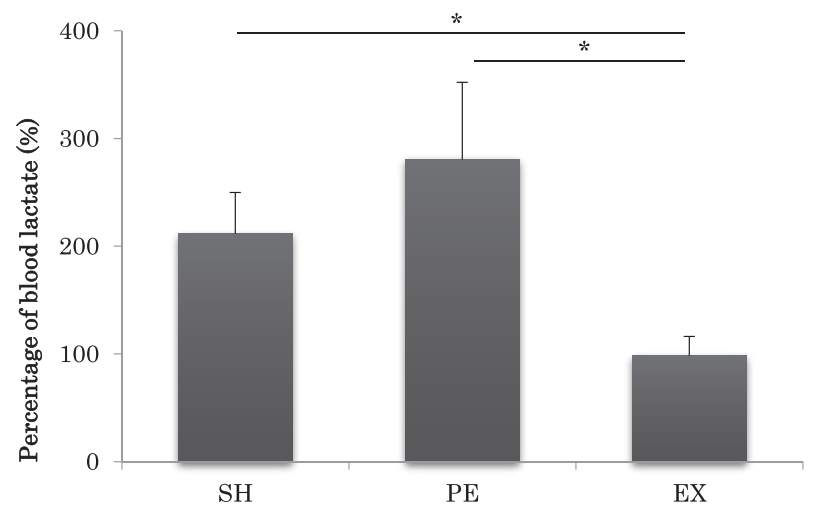

Fig. 2 Changes in blood lactate level before and after the exercise tolerance on Day 29. All data are shown as means \pm standard deviations. The blood lactate level after exercise on Day 29 significantly increased (by over $200 \%$ ) in the $\mathrm{SH}$ and $\mathrm{PE}$ groups compared with that before exercise. However, the blood lactate level in the EX group after exercise did not change compared with that before exercise.

\section{RESULTS}

Changes in blood lactate level before and after the exercise tolerance

In all animals, blood lactate levels were measured on Day 29 before and after the exercise test to determine exercise tolerance. The blood lactate levels ranged from 1.1 to $1.4 \mathrm{mmol} / \mathrm{L}$ before exercise $(\mathrm{SH}$ $1.4 \pm 0.3, \mathrm{PE} 1.2 \pm 0.3, \mathrm{EX} 1.1 \pm 0.2 \mathrm{mmol} / \mathrm{L})$. After treadmill running $(20 \mathrm{~m} / \mathrm{min}$ for $30 \mathrm{~min})$, the blood lactate level dramatically increased by over $200 \%$ in the $\mathrm{SH}$ and $\mathrm{PE}$ groups (SH group, $2.9 \pm 0.7$, representing an increase of $207 \%$; PE group $3.2 \pm 0.3$, representing an increase of $267 \%$ ) compared with before running. However, the blood lactate level in the EX group after treadmill running did not change compared with that before running $(1.0 \pm 0.2$ : $98 \%$ of the original). As a result, blood lactate levels were significantly higher in the $\mathrm{SH}$ and PE groups than in the EX group (Fig. 2). This result indicates that blood lactate levels have not changed in the EX group because exercise tolerance was enhanced by treadmill exercise for 2 weeks.

\section{Changes in respiratory function}

Tidal volume and inspiratory-expiratory ratio were calculated to examine the effect of respiratory function on the emphysema model. Using a pressure gradient transducer and respiratory flow meter, respiratory flow waveforms were recorded on Day 29, and the tidal volume was determined from the respiratory waveform. The tidal volume did not significantly differ among the three groups ( $\mathrm{SH}$ group,

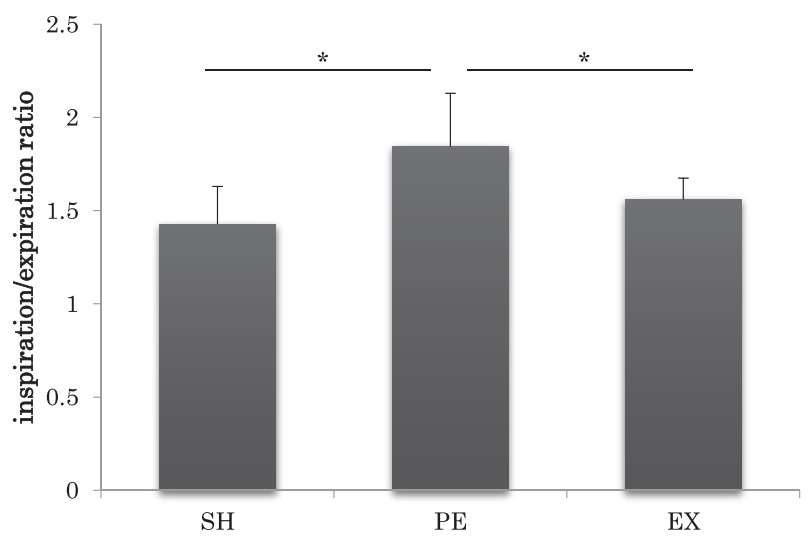

Fig. 3 Changes in inspiration/expiration ratio in each group. All data are shown as means \pm standard deviations. The inspiration/expiration ratio, calculated from the respiratory waveform, was significantly higher in the PE group than that in the $\mathrm{SH}$ and EX groups $(P<0.05)$.

$1.2 \pm 0.3 \mathrm{~mL}$; PE group, $1.1 \pm 0.2 \mathrm{~mL}$; EX group, $1.1 \pm 0.1 \mathrm{~mL}$ ). However, the inspiration/expiration ratio calculated from the respiratory waveform was significantly higher in the PE group than in the $\mathrm{SH}$ and EX groups ( $\mathrm{SH}$ group, $1.4 \pm 0.2$; $\mathrm{PE}$ group, $1.8 \pm 0.3$; and EX group, $1.6 \pm 0.1$ ) (Fig. 3).

\section{Changes in in vitro muscle contraction force}

To examine the contractile function of skeletal muscle in each group, twitch tension was measured. The specific force of the soleus muscle was $304.3 \pm$ $76.5 \mathrm{~g} / \mathrm{cm}^{2}$ in the SH group, $314.8 \pm 114.0 \mathrm{~g} / \mathrm{cm}^{2}$ in the PE group, and $326.8 \pm 79.1 \mathrm{~g} / \mathrm{cm}^{2}$ in the EX group, but these differences were not significant. In contrast, the specific force of the EDL muscle was $433.9 \pm 52.2 \mathrm{~g} / \mathrm{cm}^{2}$ in the SH group, $414.5 \pm 138.0 \mathrm{~g} /$ $\mathrm{cm}^{2}$ in the PE group, and $586.4 \pm 112.2 \mathrm{~g} / \mathrm{cm}^{2}$ in the EX group; that in the EX group was significantly increased compared with that in the $\mathrm{SH}$ and $\mathrm{PE}$ groups. The specific force of the diaphragm muscle was $467.4 \pm 93.4 \mathrm{~g} / \mathrm{cm}^{2}$ in the SH group, $474.6 \pm$ $200.6 \mathrm{~g} / \mathrm{cm}^{2}$ in the PE group, and $665.9 \pm 173.8 \mathrm{~g} / \mathrm{cm}^{2}$ in the EX group; that in the EX group was significantly increased compared with that in the SH and PE groups. Contraction time, half relaxation time and tetanic force did not significantly differ among the three groups (Table 1).

\section{Immunohistochemical staining of lung tissue}

Infiltration of CD68-positive total macrophages was detectable in the lung tissue in all groups. The CD163 and CD206 antigens are known to be upregulated in M2-type macrophages and are considered to be M2 markers. The number of CD68-positive 
Table 1 In vitro measurement of muscle contraction

\begin{tabular}{lccc}
\hline Soleus muscle & SH & PE & EX \\
\hline Specific force $\left(\mathrm{g} / \mathrm{cm}^{2}\right)$ & $304.3 \pm 76.5$ & $314.8 \pm 114.0$ & $326.8 \pm 79.1$ \\
Contraction time $(\mathrm{ms})$ & $42.8 \pm 4.0$ & $46.3 \pm 5.9$ & $46.5 \pm 4.4$ \\
Half relaxation time $(\mathrm{ms})$ & $40.2 \pm 3.1$ & $36.7 \pm 7.4$ & $40.3 \pm 6.4$ \\
Tetanic force $\left(\mathrm{g} / \mathrm{cm}^{2}\right)$ & $1197.6 \pm 239.3$ & $1209.5 \pm 144.2$ & $1182.1 \pm 178.0$ \\
\hline & & & \\
\hline Extensor digitorum longus muscle & $\mathrm{SH}$ & $\mathrm{PE}$ & $\mathrm{EX}$ \\
\hline Specific force $\left(\mathrm{g} / \mathrm{cm}^{2}\right)$ & $433.9 \pm 52.2$ & $414.5 \pm 138.0$ & $\mathbf{5 8 6 . 4} \pm \mathbf{1 1 2 . 2} * \dagger$ \\
Contraction time $(\mathrm{ms})$ & $28.5 \pm 3.7$ & $25.9 \pm 3.3$ & $30.3 \pm 4.9$ \\
Half relaxation time $(\mathrm{ms})$ & $26.6 \pm 14.2$ & $16.8 \pm 10.7$ & $22.2 \pm 10.4$ \\
Tetanic force $\left(\mathrm{g} / \mathrm{cm}^{2}\right)$ & $1425.0 \pm 136.8$ & $1286.9 \pm 167.2$ & $1395.1 \pm 57.4$ \\
\hline & \multicolumn{3}{c}{} \\
\hline Diaphragm muscle & $\mathrm{SH}$ & $\mathrm{PE}$ & $\mathrm{EX}$ \\
\hline Specific force $\left(\mathrm{g} / \mathrm{cm}^{2}\right)$ & $467.4 \pm 93.4$ & $474.6 \pm 200.6$ & $\mathbf{6 6 5 . 9} \pm \mathbf{1 7 3 . 8} \boldsymbol{*} \dagger$ \\
Contraction time $(\mathrm{ms})$ & $31.7 \pm 4.5$ & $31.2 \pm 3.3$ & $32.7 \pm 5.9$ \\
Half relaxation time $(\mathrm{ms})$ & $24.8 \pm 9.3$ & $19.9 \pm 2.3$ & $27.2 \pm 11.1$ \\
Tetanic force $\left(\mathrm{g} / \mathrm{cm}^{2}\right)$ & $975.0 \pm 141.5$ & $1018.7 \pm 420.0$ & $1269.0 \pm 283.3$ \\
\hline
\end{tabular}

All data are shown as mean \pm SD. * Significantly different from PE group $(P<0.05)$. $\dagger$ Significantly different from $\mathrm{SH}$ group $(P<0.05)$. SH, Sham group; PE, pulmonary emphysema group; EX, emphysema + exercise group.

cells per $\mathrm{mm}^{2}$ significantly differed among the three groups (SH group, 184.9 \pm 53.8 ; PE group, 690.2 \pm 196.2; EX group, $272.9 \pm 91.7)$. In addition, the infiltration of CD68- and CD206-double positive M2 macrophages significantly differed among the three groups (SH group, $32.8 \pm 9.3$; PE group, 255.6 \pm 90.5; EX group, $95.0 \pm 54.2$ ). Infiltration of CD68and CD163-double positive M2 macrophages also significantly differed among the three groups $(\mathrm{SH}$ group, $101.5 \pm 36.3$; PE group, $381.6 \pm 109.5$; EX group, $145.7 \pm 64.6)$ (Fig. 4).

Changes in serum cytokine expression before and after exercise

Serum cytokine levels were examined to determine if the inflammatory findings were systemic or local. We then evaluated the percentage of serum cytokine expression, when serum cytokine before exercise on Day 29 was regarded as $100 \%$. Interleukin- $1 \beta$ (IL$1 \beta)$, a pro-inflammatory cytokine, was $96.6 \pm 20.6 \%$ in the $\mathrm{SH}$ group, $125.7 \pm 40.8 \%$ in the PE group, and $94.7 \pm 21.5 \%$ in the EX group. IL- 6 and TNF- $\alpha$ showed similar changes (IL-6: SH group, $97.4 \pm$ $26.5 \%$; PE group, $112.5 \pm 38.7 \%$; EX group, $100.2 \pm$ $21.9 \%$ ) (TNF- $\alpha$ : SH group, $104.2 \pm 31.6 \%$; PE group, $118.8 \pm 13.1 \%$; EX group, $105.6 \pm 26.5 \%$ ). Additionally, IL-4, an anti-inflammatory cytokine, was $91.6 \pm 8.4 \%$ in the $\mathrm{SH}$ group, $115.4 \pm 41.1 \%$ in the PE group, and $93.6 \pm 17.2 \%$ in the EX group. IL-10 also showed similar changes (SH group, 93.0 \pm $7.0 \%$; PE group, $117.0 \pm 37.4 \%$; EX group $93.9 \pm$
$14.3 \%)$. Thus, there was no significant difference among the groups for all serum cytokine expression (Fig. 5).

\section{DISCUSSION}

In the present study, an emphysema model was induced by administration of cigarette smoke solution (CSS) and LPS. Fuchikami et al. reported results from a COPD model prepared by similar methods: their COPD model had infiltration of a more significant number of macrophages and neutrophils, hyperplasia of the tracheal epithelium, and rupture of the alveoli (9). In the present study, the appropriateintensity treadmill exercise load was set in the pulmonary emphysema group. Based on the exercise load, our treadmill exercise for 2 weeks improved exercise tolerance and muscle function. Additionally, the present study demonstrated that the infiltration of macrophages in the lung tissue decreased following the exercise.

Determination of appropriate-intensity treadmill running

To determine the level of treadmill running, blood lactate levels were measured immediately before exercise and at 10- and 20-min intervals during exercise. Based on the blood lactate level, we estimated that appropriate-intensity treadmill running was $17 \mathrm{~m} /$ min for $30 \mathrm{~min}$. Ni et al. (25) studied low-intensity running $(15.2 \mathrm{~m} / \mathrm{min})$, medium-intensity running 

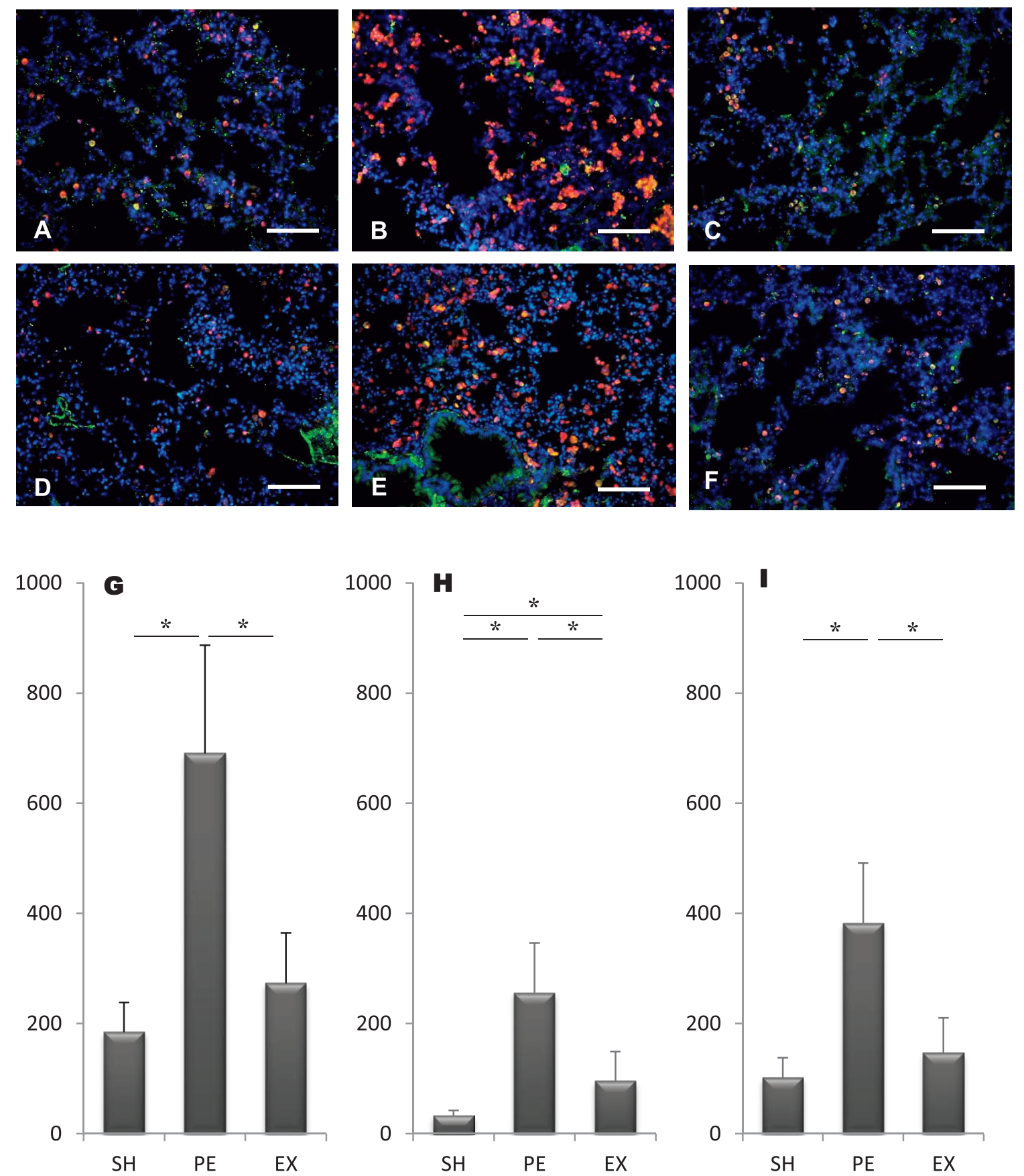

Fig. 4 Immunofluorescence staining images of macrophages in lung tissue of each group. Representative immunofluorescence staining for CD68 (red; A-F) with CD206 (green; A-C) or CD163 (green; D-E) in the SH group (A, D), PE group (B, E) and EX group (C, F). The number of CD68 ${ }^{+} / \mathrm{DAPI}^{+}$nuclei per $\mathrm{mm}^{2}(\mathrm{G}), \mathrm{CD}^{+} 8^{+} / \mathrm{CD} 206^{+} / \mathrm{DAPI}^{+}$nuclei per $\mathrm{mm}^{2}(\mathrm{H})$, and $\mathrm{CD}^{+} / \mathrm{CD} 163^{+} / \mathrm{DAPI}^{+}$nuclei per $\mathrm{mm}^{2}$ (I). All data are shown as means \pm standard deviations. Three sections per animal and 5 randomly chosen fields per section were evaluated. Scale bar $=100 \mu \mathrm{m}$. The numbers of $\mathrm{CD}^{+} 8^{+}, \mathrm{CD}^{2} 8^{+} / \mathrm{CD}^{206} 6^{+}$, and $\mathrm{CD}^{+} 8^{+} / \mathrm{CD}_{163}{ }^{+}$macrophages were significantly different among the three groups $(P<0.05)$.

$(19.3 \mathrm{~m} / \mathrm{min})$, and high-intensity running $(26.8 \mathrm{~m} /$ min) in Wistar rats and analyzed changes in knee cartilage over a training period of eight weeks. They reported that moderate-intensity exercise led to an increase in the number of cartilage cells, and strenuous-intensity exercise led a decrease in cartilage thickness and the number of cartilage cells. Bedford et al. (2) reported that $75 \% \mathrm{VO} 2 \mathrm{max}$ training im- 

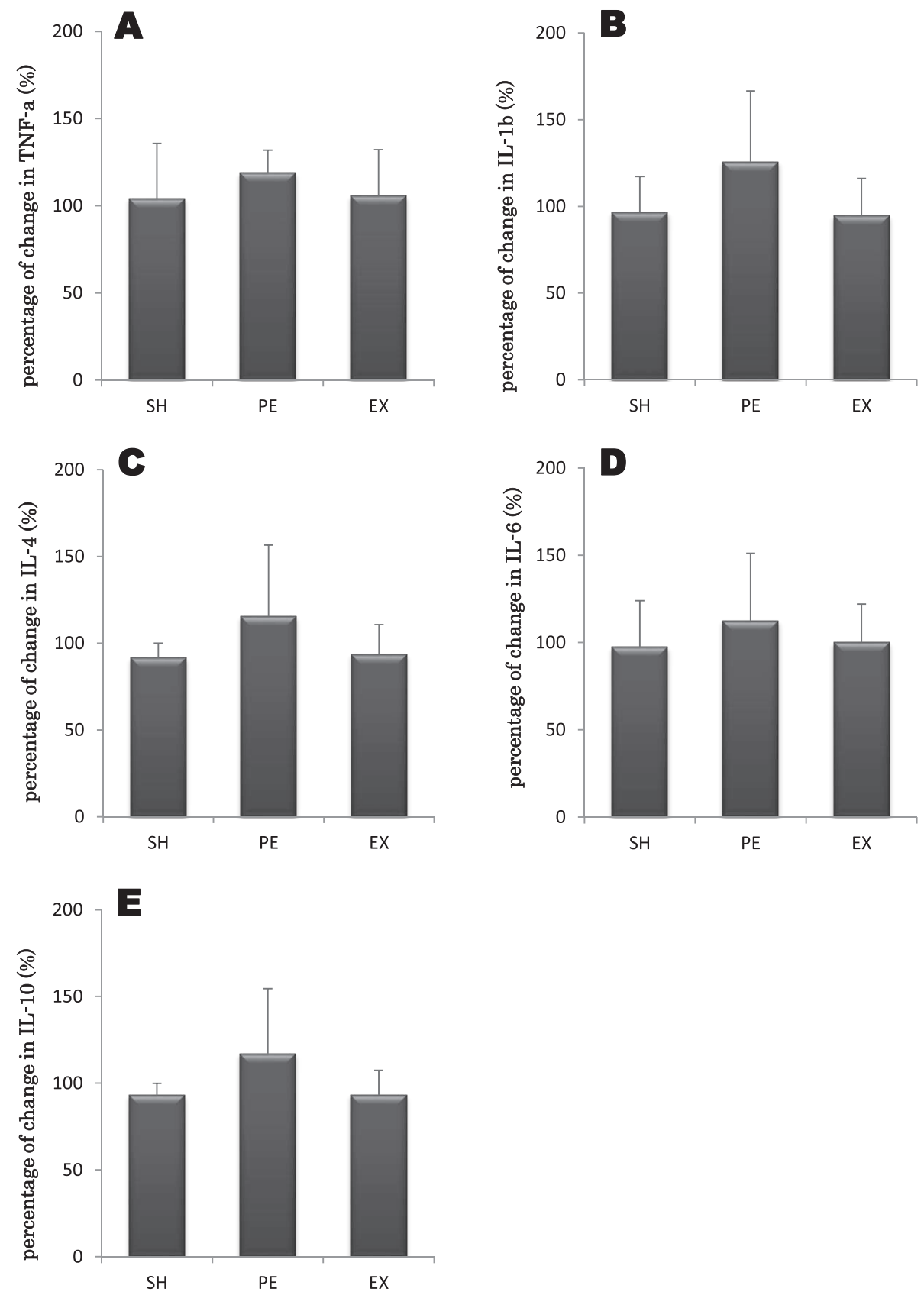

Fig. 5 Changes in serum cytokine expression before and after exercise in each group. The percentages of serum tumor necrosis factor (TNF)- $a(A)$, interleukin (IL)-1 $\beta$ (B), IL-4 (C), IL-6 (D) and IL-10 (E) in the PE group were higher than those in the other two groups. However, there was no significant difference among the three groups for expression of serum cytokines. All data are shown as means \pm standard deviations.

proved the highest performance compared between several treadmill programs and $\mathrm{VO}_{2}$. Thus, we considered that $17 \mathrm{~m} / \mathrm{min}$ was an appropriate target intensity, although the loading dose of the present study was lower than that of previous reports because the high-intensity exercise can cause inflammation and breathing difficulties in the emphysema model.

Change in skeletal muscle function by appropriateintensity treadmill running in a rat model of emphysema

Appropriate-intensity treadmill running was carried out for 2 weeks, while the emphysema model was 
developed for over 4 weeks. Blood lactate levels before and after running on Day 29 significantly differed between the EX group and the other two groups. Exercise tolerance in the EX group improved with treadmill running for 2 weeks, and this was thought to be a training effect on general endurance in the emphysema model. With respect to the contractile properties of the skeletal muscle, the specific force in the soleus muscle was not significantly different among groups, but the EDL muscle and diaphragm muscle showed significant increases in the EX group compared with those in the $\mathrm{SH}$ and $\mathrm{PE}$ groups 2 weeks after exercise. The beneficial effect of exercise in COPD patients has been shown in several clinical studies $(3,24)$. Here, the recovery of muscle function was expected following appropriate-intensity treadmill running. Moreover, we considered that training effects would occur not only for muscle function but also with respect to wholebody endurance following long-term training.

\section{Change in respiratory function by appropriate-inten- sity treadmill running}

There was no significant difference among the three groups for the tidal volume measured with a pressure gradient transducer and respiratory flow meter on Day 29 (data not shown). However, the inspiration/expiration ratio, calculated from the respiratory waveform, was significantly higher in the PE group than in the $\mathrm{SH}$ and $\mathrm{EX}$ groups. It was thought that the extension of the expiration time was induced by airway inflammation and airflow obstruction, such as from the induced emphysema by atomized administration of CSS and LPS solutions in the PE group. Several clinical studies have indicated that COPD patient's motor function and sensation of dyspnea improve by continuing exercise. We considered that although the EX group was administered CSS and LPS solution as was the PE group, the EX group recovered to levels near those of the $\mathrm{SH}$ group because their symptoms improved due to exercise training.

\section{Changes in lung tissue inflammation by appropriate- intensity treadmill running}

The number of CD68-positive macrophages in the lung tissue significantly differed among the three groups. Moreover, the number of CD68/CD206-double positive cells and the number of CD68/CD163double positive cells were significantly increased in the order of the PE group, the EX group, and the SH group. CD163 and CD206 antigens are known to be upregulated in M2-type macrophages and are considered as M2 markers. The M1 macrophage takes part in whole-body, chronic inflammation related to obesity and insulin resistance $(4,18,27)$, and the M2 macrophage takes part in the organization of chronic inflammation with an organizational change, such as $\operatorname{COPD}(12,14,30)$ and chronic nephritis (15). CD163 (macrophage scavenger receptor class A) is a receptor of the hemoglobin/haptoglobin complex, although recent studies have demonstrated that the receptor binds many ligands, such as bacteria. CD206 (macrophage mannose receptor, C type I) is a receptor involved in phagocytosis of bacteria and fungi. The mechanisms and roles of CD163 and CD206 in macrophage phenotypic changes have been unclear (16).

In our research, the remarkable inflammation of the pulmonary tissue of the PE group was drastically decreased in the EX group, suggesting that appropriate-intensity treadmill running may control the inflammatory reaction in the pulmonary tissue. With respect to cytokines measured from the serum sample, proinflammatory IL- $1 \beta$, IL-6, and TNF- $\alpha$ levels did not change in the EX and SH groups but tended to be higher in the PE group after 90 min of running on Day 29. Exercise causes a biphasic increase in the number of neutrophils in the blood and reduces monocyte numbers and the production of atherogenic cytokines, such as IFN- $\gamma$, TNF- $\alpha$, and IL- $1 \alpha$ (1, 28). Moreover, Toledo et al. reported that aerobic physical training has a protective effect on the imbalance between oxidants and antioxidants involved in the pathogenesis of cigarette smoke-induced pulmonary disease (33). In our study, although the percentage of proinflammatory cytokines (IL-1b, IL-6, and TNF- $\alpha$ ) and the number of macrophages were increased in the PE group, these proinflammatory markers were inhibited by treadmill running in the EX group (Figs. 4, 5). We speculated that appropriate-intensity treadmill running may enhance neutrophil respiratory burst activity and improvement of the circulatory dynamics in respiratory organs.

In general, emphysema damages the inner walls of the lungs' air sacs (alveoli), eventually causing them to rupture. This creates one larger air space instead of many smaller ones and reduces the surface area available for gas exchange. In our research, because only a short period of time was needed to induce the emphysema model, damage to the pulmonary alveolus may have created an imperfect model of emphysema. 


\section{Acknowledgements}

We thank Keisuke Okada and Tadayuki Mita for expert technical assistance. This research was supported in part by Grants-in-Aid for Scientific Research (C) from the Japan Society for the Promotion of Science (24500628 and 17K01547) and Kio University Fellowship for Scholars and Researchers to Project Research.

\section{REFERENCES}

1. Beavers KM, Brinkley TE and Nicklas BJ (2010) Effect of exercise training on chronic inflammation. Clin Chim Acta 411, 785-793.

2. Bedford TG, Tipton CM, Wilson NC, Oppliger RA and Gisolfi CV (1979) Maximum oxygen consumption of rats and its changes with various experimental procedures. $J$ Appl Physiol 47, 1278-1283.

3. Bernardi E, Pomidori L, Bassal F, Contoli M and Cogo A (2015) Respiratory muscle training with normocapnic hyperpnea improves ventilatory pattern and thoracoabdominal coordination, and reduces oxygen desaturation during endurance exercise testing in COPD patients. Int J Chron Obstruct Pulmon Dis 10, 1899-1906.

4. Bouhlel MA, Derudas B, Rigamonti E, Dievart R, Brozek J, et al. (2007) PPARgamma activation primes human monocytes into alternative M2 macrophages with anti-inflammatory properties. Cell Metab 6, 137-143.

5. Carr-Lopez S, Salem H and Catania PN (1998) Medication use in home care patients with COPD. Home Care Provid 3, 144-148.

6. Clini EM and Ambrosino N (2008) Nonpharmacological treatment and relief of symptoms in COPD. Eur Respir J 32, 218-228.

7. Crowley TJ, Macdonald MJ and Walter MI (1995) Behavioral anti-smoking trial in chronic obstructive pulmonary disease patients. Psychopharmacology (Berl) 119, 193-204.

8. Flynn RA, Glynn DA and Kennedy MP (2009) Anticholinergic treatment in airways diseases. Adv Ther 26, 908-919.

9. Fuchikami J and Takahashi M (2006) Cigarette smokeinduced animal models to evaluate drug efficacy on chronic obstructive pulmonary disease (COPD). Nihon Yakurigaku Zasshi 127, 183-189.

10. Fukuchi Y, Nishimura M, Ichinose M, Adachi M, Nagai A, et al. (2004) COPD in Japan: the Nippon COPD Epidemiology study. Respirology 9, 458-465.

11. Ghanem M, Elaal EA, Mehany M and Tolba K (2010) Home-based pulmonary rehabilitation program: Effect on exercise tolerance and quality of life in chronic obstructive pulmonary disease patients. Ann Thorac Med 5, 18-25.

12. Graff JW, Powers LS, Dickson AM, Kim J, Reisetter AC, et al. (2012) Cigarette smoking decreases global microRNA expression in human alveolar macrophages. PLoS One 7, e44066.

13. Hess MW (2017) The 2017 Global Initiative for Chronic Obstructive Lung Disease Report and Practice Implications for the Respiratory Therapist. Respir Care 62, 1492-1500.

14. Hodge S, Matthews G, Mukaro V, Ahern J, Shivam A, et al. (2011) Cigarette smoke-induced changes to alveolar macrophage phenotype and function are improved by treatment with procysteine. Am J Respir Cell Mol Biol 44, 673-681.
15. Ikezumi Y, Suzuki T, Karasawa T, Hasegawa H, Yamada T, et al. (2011) Identification of alternatively activated macrophages in new-onset paediatric and adult immunoglobulin A nephropathy: potential role in mesangial matrix expansion. Histopathology 58, 198-210.

16. Kaku Y, Imaoka H, Morimatsu Y, Komohara Y, Ohnishi K, et al. (2014) Overexpression of CD163, CD204 and CD206 on alveolar macrophages in the lungs of patients with severe chronic obstructive pulmonary disease. PLoS One 9, e87400.

17. Katsura H, Kanemaru A, Yamada K, Motegi T, Wakabayashi $\mathrm{R}$, et al. (2004) Long-term effectiveness of an inpatient pulmonary rehabilitation program for elderly COPD patients: comparison between young-elderly and old-elderly groups. Respirology 9, 230-236.

18. Kitade H, Sawamoto K, Nagashimada M, Inoue H, Yamamoto Y, et al. (2012) CCR5 plays a critical role in obesity-induced adipose tissue inflammation and insulin resistance by regulating both macrophage recruitment and M1/M2 status. Diabetes 61, 1680-1690.

19. Kizaki T, Takemasa T, Sakurai T, Izawa T, Hanawa T, et al. (2008) Adaptation of macrophages to exercise training improves innate immunity. Biochem Biophys Res Commun 372, $152-156$

20. Landbo C, Prescott E, Lange P, Vestbo J and Almdal TP (1999) Prognostic value of nutritional status in chronic obstructive pulmonary disease. Am J Respir Crit Care Med 160, 18561861.

21. MacIntyre NR (2008) Mechanisms of functional loss in patients with chronic lung disease. Respir Care 53, 1177-1184.

22. Mizutani N, Fuchikami J, Takahashi M, Nabe T, Yoshino S, et al. (2009) Pulmonary emphysema induced by cigarette smoke solution and lipopolysaccharide in guinea pigs. Biol Pharm Bull 32, 1559-1564.

23. Montuschi P (2006) Pharmacological treatment of chronic obstructive pulmonary disease. Int J Chron Obstruct Pulmon Dis 1, 409-423.

24. Mussi RK, Camargo EA, Ferreira T, De Moraes C, Delbin MA, et al. (2008) Exercise training reduces pulmonary ischaemia-reperfusion-induced inflammatory responses. Eur Respir J 31, 645-649.

25. Ni GX, Liu SY, Lei L, Li Z, Zhou YZ, et al. (2013) Intensity-dependent effect of treadmill running on knee articular cartilage in a rat model. Biomed Res Int 2013, 172392.

26. Nieman DC (2007) Marathon training and immune function. Sports Med 37, 412-415.

27. Olefsky JM and Glass CK (2010) Macrophages, inflammation, and insulin resistance. Аnnu Rev Physiol 72, 219-246.

28. Peake JM (2002) Exercise-induced alterations in neutrophil degranulation and respiratory burst activity: possible mechanisms of action. Exerc Immunol Rev 8, 49-100.

29. Reardon JZ, Lareau SC and ZuWallack R (2006) Functional status and quality of life in chronic obstructive pulmonary disease. Am J Med 119, 32-37.

30. Shaykhiev R, Krause A, Salit J, Strulovici-Barel Y, Harvey $\mathrm{BG}$, et al. (2009) Smoking-dependent reprogramming of alveolar macrophage polarization: implication for pathogenesis of chronic obstructive pulmonary disease. J Immunol 183, 28672883.

31. Suzuki K, Nakaji S, Yamada M, Liu Q, Kurakake S, et al. (2003) Impact of a competitive marathon race on systemic cytokine and neutrophil responses. Med Sci Sports Exerc 35, 348-355.

32. Theander K, Jakobsson P, Jorgensen N and Unosson M (2009) Effects of pulmonary rehabilitation on fatigue, functional sta- 
tus and health perceptions in patients with chronic obstructive pulmonary disease: a randomized controlled trial. Clin Rehabil 23, 125-136.

33. Toledo AC, Magalhaes RM, Hizume DC, Vieira RP, Biselli PJ, et al. (2012) Aerobic exercise attenuates pulmonary injury induced by exposure to cigarette smoke. Eur Respir $J \mathbf{3 9}$, 254-264.

34. Tsara V, Serasli E, Katsarou Z, Tsorova A and Christaki P (2008) Quality of life and social-economic characteristics of greek male patients on long-term oxygen therapy. Respir Care
53, 1048-1053.

35. Wouters EF, Reynaert NL, Dentener MA and Vernooy JH (2009) Systemic and local inflammation in asthma and chronic obstructive pulmonary disease: is there a connection? Proc Am Thorac Soc 6, 638-647.

36. Yoneda T, Yoshikawa M, Fu A, Tsukaguchi K, Okamoto Y, et al. (2001) Plasma levels of amino acids and hypermetabolism in patients with chronic obstructive pulmonary disease. Nutrition 17, 95-99. 